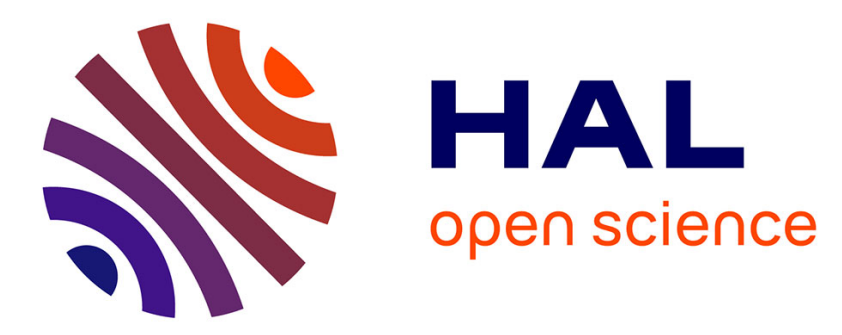

\title{
Generalization of the interaction between the Haar approximation and polynomial operators to higher order methods
}

François Chaplais

\section{- To cite this version:}

François Chaplais. Generalization of the interaction between the Haar approximation and polynomial operators to higher order methods. Analysis in Theory and Applications, 2006, pp.301-318. 10.1007/s10496-006-0301-0 . hal-00492442

HAL Id: hal-00492442

https://hal-mines-paristech.archives-ouvertes.fr/hal-00492442

Submitted on 15 Jun 2010

HAL is a multi-disciplinary open access archive for the deposit and dissemination of scientific research documents, whether they are published or not. The documents may come from teaching and research institutions in France or abroad, or from public or private research centers.
L'archive ouverte pluridisciplinaire HAL, est destinée au dépôt et à la diffusion de documents scientifiques de niveau recherche, publiés ou non, émanant des établissements d'enseignement et de recherche français ou étrangers, des laboratoires publics ou privés. 


\title{
Generalization of the interaction between the Haar approximation and polynomial operators to higher order methods
}

\author{
François Chaplais \\ Centre Automatique et Systèmes \\ École Nationale Supérieure des Mines de Paris \\ 35 rue Saint-Honoré 77305 Fontainebleau CEDEX FRANCE \\ chaplais@cas.ensmp.fr
}

December 13, 2006

\begin{abstract}
In applications it is useful to compute the local average of a function $f(u)$ of an input $u$ from empirical statistics on $u$. A very simple relation exists when the local averages are given by a Haar approximation. The question is to know if it holds for higher order approximation methods. To do so, it is necessary to use approximate product operators defined over linear approximation spaces. These products are characterized by a Strang and Fix like condition. An explicit construction of these product operators is exhibited for piecewise polynomial functions, using Hermite interpolation. The averaging relation which holds for the Haar approximation is then recovered when the product is defined by a two point Hermite interpolation.
\end{abstract}

Keywords Strang and Fix conditions Product Approximation Hermite Interpolation Wavelets

\section{Introduction}

The theory of averaging [1] approximates the solutions of a differential systems $d x / d t=f(x, t, t / \epsilon)$, where $\epsilon$ is a small parameter, by the solution of an 
"averaged" system $d x / d t=\bar{f}(x, t)$. In practice, $f$ is not given as a function of two time scales $t$ and $t / \epsilon$, but rather as a function $f(x, u)$ where $u$ is an input which is a function of time. The question is to give a practical sense to the "average" of $f(x, u)$ as a function of the input $u$.

The answer is relatively simple when the averaging of $f$ (and $u$ ) is obtained by computing its averages on a sequence of intervals $[k \delta,(k+1) \delta)$. In this paper, we assume that $f$ is a polynomial function. If $E$ denotes the averaging operator, it can be easily shown that the following identity holds:

$$
E(f(u))=\sum_{k=0}^{k=\infty} \frac{f^{(k)}(e)}{k !} E\left(w^{k}\right)
$$

where $e=E(u)$ and $w=u-e$. We can see that $f$ is expanded around the average of $u$, and the powers of $w$ are replaced in the expansion by their averages. If one interprets the averaging operator $E$ as an empirical expectation, then (1) shows that the expectation of $f(u)$ is computed by using the derivatives of $f$ at the expectation $e$ of $u$ and the moments of the "noise" $w$. While it is easy to derive, equation (1) appears to be new or at least not a classic.

This previous method of averaging is also an approximation procedure which is related to the Haar multiresolution analysis. There are other methods of approximation which are more efficient and yet give a sense of local average. Wavelets $[2,3,4]$ provide such methods. The order of approximation are then given by the classic Strang and Fix conditions [5] which states that the order of approximation of smooth functions is characterized by the ability of the approximation method to reproduce polynomials up to a certain order. This condition is related to the number of vanishing moments of the wavelet. The approximation of a function is obtained by projection on a resolution space, which is itself generated by the translates of a so called scaling function. The previous averaging is a particular case of approximation on a resolution space where the scaling function is the characteristic function of an interval. The corresponding multiresolution analysis is called the Haar system. Among all wavelet methods, it yields the lowest order of approximation, since its scaling function reproduces only polynomials of degree zero.

The following question then arises: is it possible to have the relation (1) for higher order approximation methods (wavelets or not)? This will be the main subject of this article. 
It turns out that projectors which satisfy equation (1) can be simply characterized by two conditions on their image space and their kernel:

Proposition 1 Let $\boldsymbol{V}$ the image space of the projector $E$ and $\boldsymbol{W}$ the image of Id-E. Equation (1) is verified if and only if

(P1) the product of two elements of $\boldsymbol{V}$ belongs to $\boldsymbol{V}$

(P2) the product of a element of $\boldsymbol{V}$ with a element of $\boldsymbol{W}$ is a element of $\boldsymbol{W}$

The sufficient part is proved by expanding $f$ around $e$ and applying $E$. The necessary condition is derived from (1) using $f(x)=x^{2}$ with $x=e+b$ and $x=e-b, e \in \mathbf{V}$ and successively $b \in \mathbf{V}$ and $b \in \mathbf{W}$.

Finding approximation projectors which satisfy equation (1) if thus equivalent to satisfying properties $(P 1)$ and $(P 2)$. As we shall see in the next section, property $(P 1)$ implies that the function whose translates generates $\mathbf{V}$ is the characteristic function of an interval. It appears then that high order approximation methods cannot satisfy property $(P 1)$.

This last limitation is due to the use of the classical product on functions. Since we are dealing with approximations of functions, we can replace the classical product by an approximate product without degrading the order of approximation of the result. A general characterization of such approximate products is given in section 2.2.2. Like the Strang and Fix conditions, it is based on the reproduction of polynomials.

There remains to construct such approximate products. The difficulty is to verify the associativity of the product operator. In the case where the functions are piecewise polynomial, we give in section 3.2 a constructive characterization of approximate products in this functional space. These products are defined by a Hermite interpolation. In the simple case of a two point interpolation, we exhibit in section 3.4 an approximation projector which satisfies properties (P1) and (P2), and thus equation (1).

\section{Product invariance and approximation}

\subsection{Two results on product invariance}

The property (P1) states that the space $\mathbf{V}$ should be product invariant. We consider here the case where $\mathbf{V}=\mathbf{V}_{\delta} \subset \mathbf{L}^{2}(\mathbb{R})$ has a Riesz basis $\left(\phi_{k}\right)_{k \in \mathbb{Z}}$ with $\left.\phi_{k}(t)=\phi(t / \delta-k) / \sqrt{(} \delta\right)$, and $\phi$ is compactly supported. In the wavelet 
context, $\mathbf{V}_{\delta}$ is a resolution space and $\phi$ is a scaling function. Given a function $e \in \mathbf{V}_{\delta}, C_{k}(e)$ will denote its coordinate on the vector $\phi_{k}$. The following general lemma characterizes the product operators for which (P1) holds:

Lemma 1 Let $*$ a (nonzero) product operator such that $\left(\boldsymbol{V}_{\delta},+, *\right)$ be a commutative ring. It is assumed that that $*$ commutes with the shift of length $\delta$, and that the product of two compactly supported functions is zero beyond some fixed distance. Then there exist a constant $A \neq 0$ such that, for any $x$ and $y$ in $\boldsymbol{V}_{\delta}$, and any $n \in \mathbb{N}$,

$$
C_{n}(x * y)=A C_{n}(x) C_{n}(y)
$$

If $*$ is the usual product on functions, then the intersection of the supports of $\phi_{i}$ and $\phi_{j}$ is of measure zero if $i \neq j$.

This lemma is proved in appendix A. Let us apply it to the case where an approximation operator is associated to the space $\mathbf{V}_{\delta}$ :

Theorem 1 Let $E_{\delta}$ a projector on $\boldsymbol{V}_{\delta}$ defined by a rescaled kernel: $E_{\delta} x(t)=$ $1 / \delta \int K(t / \delta, s / \delta) x(s) d s$. We assume that $E_{\delta}$ is an approximation operator, e.g.,for any $f \in \mathbf{L}^{2}(\mathbb{R})$,

$$
\left\|E_{\delta} f-f\right\|_{\mathbf{L}^{2}(\mathbb{R})} \rightarrow 0 \text { when } \delta \rightarrow 0 .
$$

If $\boldsymbol{V}_{1}$ is product invariant for the usual product, then $\phi$ is proportional to the characteristic function of an interval of length 1.

Proof. Thanks to the projector $E_{\delta}$, the linear form $C_{k}$ is extended to $\mathbf{L}^{\mathbf{2}}(\mathbb{R})$. Under mild assumptions, it can be represented by an $\mathbf{L}^{\mathbf{2}}(\mathbb{R})$ function $\phi_{k}^{*}$; one can easily see that $\left.\phi^{*}(t)=\phi^{*}(t / \delta-k) / \sqrt{(} \delta\right)$ with $\phi *$ compactly supported and that $K(t, s)=\phi(t) \phi^{*}(s)$. The Strang and Fix conditions [5] imply that $E(1)$ is defined and is equal to 1 . Since the $\phi_{k}$ 's have disjoint support and have the same shape, they must be proportional to the characteristic function of an interval of length 1.

Theorem 1 implies that, if $E_{\delta}$ yields an order of approximation strictly greater than the minimum provided by the Haar basis, then $\mathbf{V}_{\delta}$ cannot satisfy $(P 1)$. Indeed, $\phi$ cannot be a piecewise constant function anymore, and the only assumption that may be invalid in lemma 1 with the usual product is the product invariance of $\mathbf{V}_{\delta}$. 


\subsection{Characterization of approximate products (a gen- eralization of the Strang and Fix conditions)}

\subsubsection{Introduction}

While (2) does not hold for higher order approximations, it is not far from being verified; if $\delta$ is small, $1 / \delta \phi(t / \delta)$ is close to a Dirac, and the sample of a product is the product of the samples. In general, this product only yields a first order approximation. Coiflets $[2,6]$ provide scaling functions with a high order approximation which are good approximations of Diracs. This suggests that using approximate product operators may recover the product invariance of $\mathbf{V}$. Such approximate product operators are characterized in the next section.

\subsubsection{The polynomial condition for product approximation}

Assumptions: let $*$ a product operator on $\mathbf{V}_{1}$ such that $\left(\mathbf{V}_{1},+, *\right)$ be a commutative ring. It is assumed that that $*$ commutes with the shift of length $L, L \in \mathbb{R}$ and that is continuous and localized as follows: there exists $(K, \mu)$ such that

$$
|f * g|(t) \leq K \sup _{|s-t| \leq \mu}|f(s)| \sup _{|s-t| \leq \mu}|g(s)|
$$

The product operator $*_{\delta}$ is defined on $\mathbf{V}_{\delta}$ by rescaling: $f(t / \delta) *_{\delta} g(t / \delta)=$ $(f * g)(t / \delta)$. It is assumed that $E_{\delta}$ satisfies the Strang and Fix conditions [5] at order $N$, e.g. $E_{\delta}\left(t^{i}\right)=t^{i}$ if $i \leq N$. Then

Theorem 2 The following two conditions are equivalent

- There exists $K$ such that, for any $f$ and $g$ of class $C^{N+1}$ and any $\delta \leq 1$,

$$
\left|f g(t)-\left[\left(E_{\delta} f\right) *_{\delta}\left(E_{\delta} g\right)\right](t)\right| \leq K \delta^{N+1} \sup _{(k, l) \in I_{N},|s-t| \leq(\mu+M) \delta}\left|f^{(k)}(s)\right|\left|g^{(l)}(s)\right|
$$

where $I_{N}$ denotes the set of integers $(k, l) \in \mathbb{N} \times \mathbb{N}$ such that $0 \leq k \leq$ $N+1,0 \leq l \leq N+1$ and $k+l \geq N+1$.

- $t^{i} * t^{j}=t^{i+j}$ if $i+j \leq N$ 
The proof of this theorem is in appendix B. Observe its similarity with the classical Strang and Fix conditions for linear operators [5].

Note that Beylkin [7] has designed a recursive algorithm to compute the wavelet coefficients on the product of two functions. It converges fast if the functions are regular. The computation of the exact product involves an infinite number of computations; an approximation is computed by stopping the recursion before the infinity. The result presented here is different in the sense that it characterizes approximate product operators which are compatible with the scaling operation. In section 3 we effectively build such operators for piecewise polynomial functions.

\section{Working with piecewise polynomial func- tions}

\subsection{Introduction}

Since the mapping $x \rightarrow a \times x$ is linear, building a product operator appears at a first glance as the construction of a collection of linear operators. The extra requirement that the operator be associative shows that things are more complicate; indeed, checking the associativity is a nonlinear problem. This eliminates Fourier methods for finding approximate products.

A case where approximate products are easy to find is when the coordinate of the approximation of a function is a sample (interpolating scaling functions $[8,9])$ or an approximation of a sample (Coiflets). The approximate product is defined by having the coordinate of the product to be the product of the coordinates, as in lemma 1 . The functional space on which the general product is defined is a resolution space at a fine scale. There remains to define an approximation subspace which is product invariant. Since we are dealing with multiresolution analysis, the natural idea is to consider a coarser resolution space. In general, it is not product invariant under the action of the product which is defined on the fine scale. Take for instance the Schauder basis, where approximations are defined by linear approximations between samples. The scaling function is the hat function, which is the autocorrelation of the Haar function $\mathbf{1}_{[0,1)}$. Then the coarser resolution spaces are not product invariant. Indeed, let us consider a coarse scaling function, e.g. a scaled hat function; if the resolution space was product invariant, the square of the scaling function would be a linear interpolation over the coarse grid. This 
is not the case, because the approximate square is computed by performing an interpolation of the square over the fine grid, and the result is a linear interpolation over samples of a parabola. It is not a linear interpolation over points of the coarse grid. So, even in this simple case, the matter of finding product invariant approximation subspaces is not obvious.

The nature of approximating product invariant subspaces is linked to the choice of the approximate product operator. To better understand the nature of this link, we have chosen to restrict our attention to the special case where functions are piecewise polynomial ones. This will allow us to characterize the relationship between the choice of the operator and the approximation subalgebras.

Observe that wavelets can detect singularities in piecewise regular functions (provided the singularities are not too close) and analyze them $([10,2])$. Then these function pieces can be approximated by piecewise polynomial functions. We can therefore assume to have an approximation of piecewise regular functions as piecewise polynomials.

We now assume assume that the function has been preprocessed to be represented as an equally spaced piecewise polynomial function of degree smaller than some $N \in \mathbb{N}$. The study of conditions $(P 1)$ and $(P 2)$ will not bear on general functions but on piecewise polynomial ones.

This section is organized as follows. Section 3.2 characterizes the approximate product on piecewise polynomial functions by showing that they must be defined by a Hermite interpolation. To recover property (P1), section 3.3 studies the approximation subspaces of piecewise polynomial functions which are product invariant. In particular, all of these subspaces include a minimal one which consists in so called regular functions. Finally, section 3.4 studies the case where the approximate product is defined by a Hermite interpolation between two points and exhibits an approximation projector which satisfies properties (P1) and (P2), and hence equation (1).

\subsection{Characterization of approximate products for piece- wise polynomial approximations}

\subsubsection{The algebraic Strang and Fix conditions for piecewise poly- nomial approximations}

Denote by $S_{N, \delta}(t)$ the space of piecewise polynomial functions over successive intervals of length $\delta$ and with a degree smaller or equal to $N$. To maintain 
consistency with the linear and nonlinear approximation conditions previously mentioned, we assume there exists a linear approximation operator $\mathbf{P}_{\delta}$ which transforms functions into elements of $S_{N, \delta}(t) . \mathbf{P}_{\delta}$ is defined by scaling a kernel $K$ which is assumed to have the structure

$$
K(t, s)=\sum_{k \in \mathbb{Z}} \sum_{l=0}^{l=N}(t-k)^{l} 1_{[k, k+1)}(t) \varphi_{l}(s-k)
$$

and to satisfy the assumptions ${ }^{1}$ of the result of Strang and Fix with the order $N$ (two such operators are presented in [11]). Theorem 2 is transposed to $S_{N, \delta}(t)$ and relates it to an algebraic condition:

Proposition 2 Denote by $\mathbb{R}_{N}[t]$ the space of real valued polynomials with degree smaller or equal to $N$, and by $\boldsymbol{F}_{\delta}$ the operator $\left[\boldsymbol{F}_{\delta} p\right](t)=\sum_{k \in \mathbb{Z}} p_{k}(t / \delta-k) 1_{[k, k+1)}(t / \delta)$ which identifies polynomial sequences in $\mathbb{R}_{N}[t]$ to functions in $S_{N, \delta}(t)$. If $\times$ is a product operator over $\mathbb{R}_{N}[t]$, it is transposed to an operator $*_{\delta}$ on functions in $S_{N, \delta}(t)$ as follows:

$$
\left(f *_{\delta} g\right)(t)=\boldsymbol{F}_{\delta}\left(\left(\boldsymbol{F}_{\delta}^{-1} f\right)_{k} \times\left(\boldsymbol{F}_{\delta}^{-1} g\right)_{k}\right)_{k \in \mathbb{Z}}
$$

Then $*_{\delta}$ approximates the product on the image of $P_{\delta}$ like in condition (4) of theorem 2 if and only if $\times$ satisfies:

$$
t^{i} \times t^{j}=t^{i+j} \in \mathbb{R}_{N}[t] \text { if } i+j \leq N
$$

This result is proved in [11].

Comment: the degree of the product is restricted to be smaller or equal to $N$ in order to have $S_{N, \delta}(t)$ product invariant. This, in turns, makes sure that the complexity of the function representation does not increase with every product operation. It also makes sense numerically: since the elements of $S_{N, \delta}(t)$ are at best approximations of regular functions at the order $N$, there is no need for a greater precision on the computation of the product.

\subsubsection{Description of all approximate products}

Proposition 2 reduces the search for approximate products over $S_{N, \delta}(t)$ to algebraic products on $\mathbb{R}_{N}[t]$ which satisfy (8). These are characterized in the following lemma:

\footnotetext{
${ }^{1}$ In particular, it is defined by a shift invariant scaled kernel.
} 
Lemma 2 (Hermite interpolation product) Let $\times$ an associative, commutative product over $\mathbb{R}_{N}[t]$ which satisfies (8), and define $T_{N+1} \in \mathbb{R}_{N}[t]$ by $T_{N+1}=t^{N} \times t$. Then, for any $p$ and $q$ in $\mathbb{R}_{N}[t], p \times q$ is the Hermite interpolation of pq at the (possibly multiple) zeros of $t^{N+1}-T_{N+1}(t)$ in $\mathbb{C}$. Conversely, any such product is associative, commutative, and satisfies (8) in $\mathbb{R}_{N}[t]$.

This lemma is proved in appendix C.

Interpretation: it is convenient to understand the product operators $\times$ of lemma 2 as follows:

- at every interpolation point $z$ of order $o$, a Taylor expansion at order $o$ of the polynomials $x$ and $y$ is computed;

- at every interpolation point $z$ of order $o$, the product of the expansions is computed and the coefficients of degree greater than $o$ are set to zero; denote by $p_{z}$ the result;

- the product $x \times y$ is then the Hermite interpolation of the various $p_{z}$.

This leads to the following constructive characterization of approximate product operators on $S_{N, \delta}(t)$

Theorem 3 Let $*_{\delta}$ a product operator on $S_{N, \delta}(t)$ related to an algebraic product operator $\times$ by (7). Then $*_{\delta}$ approximates the product on the image of $P_{\delta}$ like in condition (4) of theorem 2 if and only $p \times q$ is defined by the Hermite interpolation of $p q$ in $\mathbb{R}_{N}[t]$ on a set of interpolation points.

Examples Assume that $N$ is odd, $N=2 p+1$. Then the product can be defined as the Hermite interpolation of the product at the points 0 and 1 and orders $p$. For $p=0$, the product is the linear interpolation of product of the samples. For continuous functions, the resulting product is the same as the product previously exhibited on the Schauder basis.

\subsection{Product invariant approximation subspaces}

Our working space is now $S_{N, \delta}(t)$. If we remember the decomposition $x=$ $e+w, e$ should belong to an approximation space which satisfies condition (P1), e.g. it is product invariant. This section shows that all such approximation spaces include a minimal algebra which is defined by "regular" functions. In particular, the number of parameters required per time step to 
describe a regular function has a non zero lower bound. This is a contrast to the situation of multiresolution approximations where a nested structure of approximation spaces exist such that the number of parameters per time unit required to describe functions in a resolution space tends to zero as the scale tends to the infinity.

\subsubsection{A variation on the Strang and Fix condition}

All of the previous results require the existence of an approximation operator for regular functions. Consistently, we characterize the linear operators $\mathbf{Q}_{\delta}$ on $S_{N, \delta}(t)$ which approximate regular functions at the order $N$. Consistently again, we assume that $\mathbf{Q}_{\delta}=\mathbf{F}_{\delta} \mathbf{Q} \mathbf{F}_{\delta}^{-1}$, where $\mathbf{Q}$ applies a unique operator $Q$ on blocks of $q$ successive polynomials. In other words, $\mathbf{Q}_{\delta}$ is defined by q-shifting and scaling an operator $Q$ on $\left(\mathbb{R}_{N}[t]\right)^{q}$.

Proposition 3 Let $\boldsymbol{P}_{\delta}$ the scaled operator which codes functions into piecewise polynomials. Then $\boldsymbol{Q}_{\delta} \boldsymbol{P}_{\delta}$ approximates functions of class $C^{N+1}$ with an error of order $\delta^{N+1}$ if and only if

$$
Q\left[\left((t+k)^{p}\right)_{k \in \mathbb{Z}}\right]=\left((t+k)^{p}\right)_{k \in \mathbb{Z}} \text { for } 0 \leq p \leq N
$$

The proof essentially consists in showing that $\mathbf{Q}_{\delta} \mathbf{P}_{\delta}$ has the structure required by the result of Strang and Fix with a shift length $q \delta$. Details of the proof are in [11]. The choice of $q>1$ is to allow more general operations on piecewise polynomials like combined interpolation and subsampling.

In looking for subspaces of $S_{N, \delta}(t)$, we will require that they be defined as the image of an approximation operator $\mathbf{Q}_{\delta}$. An important consequence of the previous proposition is that these subspaces must contain the functions $t^{i}, i \leq N$; if they are product invariant, they must contain all the monomials defined by the approximate product operator.

\subsubsection{Product invariant subspaces and Hermite interpolation points}

We have characterized approximate product operators over $S_{N, \delta}(t)$. To define an approximation on this space which satisfies property $(P 1)$, we must study the subspaces of $S_{N, \delta}(t)$ which are product invariant and include polynomials of degree smaller or equal to $N$, a condition which is necessary for them to qualify as an approximation spaces. It turns out that all of these various spaces include a minimal one, which is the space of so-called regular functions of $S_{N}$. 
Notations Let $\times$ an associative, commutative product over $\mathbb{R}_{N}[t]$ which is defined by a Hermite interpolation as in theorem 2 .

- $\mathbf{Z}$ is the set of interpolation points which defines the product $\times$, without counting the order of multiplicity.

- if $z \in \mathbf{Z}, o(z)$ denotes the order of interpolation used by the product at the point $z$.

- $I$ denotes an arbitrary finite subset of $I$ and $S_{N, I}[t]$ is the space of sequences with elements in $\mathbb{R}_{N}[t]$ and indices in $I$.

\section{Regular functions}

Definition 1 (Regular functions) The subspace $\tilde{S}_{N, I}[t]$ of "regular functions" in $S_{N, I}[t]$ is the set of sequences $p \in S_{N, I}[t]$ such that $p_{k}^{(j)}(z)=p_{\tilde{k}}^{(j)}(\tilde{z})$ if $z+k=\tilde{z}+\tilde{k}$ and $j<\min (o(z), o(\tilde{z}))$.

Interpretation: sampling points $z$ and $\tilde{z}$ such that $z+k=\tilde{z}+\tilde{k}$ with $k, \tilde{k} \in \mathbb{Z}$ are called overlapping sampling points. The redundancy introduced by the regularity can be removed by identifying any regular function to a unique polynomial, while preserving the product. At every sampling point, for all indices, the maximum order of interpolation is computed for all sampling points which may overlap here. The global polynomial is defined by Hermite interpolation at these maximum orders. The polynomial product is defined by the same interpolation. This establishes an algebraic isomorphism between regular functions and a single polynomial algebra. The dimension of the latter is $\Omega(I)$, which is the sum of all the orders of interpolation increased by one $^{2}$. An important property of $\Omega(I)$ is that it is greater or equal to $N$ plus the number of elements of $I$, if $I$ is finite. Indeed, $\Omega(I)$ is an increasing function of this number of elements, since each shift of the interpolation points adds at least one node which does not overlap the previous ones. If $I$ has one element, then $\Omega(I)=N+1$.

Minimum approximation subalgebras Regular functions are the minimum approximation product invariant subspace of piecewise polynomials:

\footnotetext{
${ }^{2}$ At an interpolation point, the number of parameters describing the function is equal to the order plus 1
} 
Theorem 4 (Minimum subalgebra) - $\tilde{S}_{N, I}[t]$ is a product invariant subspace of $S_{N, I}[t]$ which includes the sequences $\left((t+k)^{p}\right)_{k \in I}$ for $0 \leq$ $p \leq N$.

- If $I$ is finite and if $\Sigma_{I}$ is a subalgebra of $S_{N, I}[t]$ which includes the sequences $\left((t+k)^{p}\right)_{k \in I}$ for $p=0$ and $p=1$, then $\tilde{S}_{N, I}[t] \subset \Sigma_{I}$.

Proof. Let us prove the first statement. From the definition of $\tilde{S}_{N, I}[t]$ and the definition of the product, it is easy to verify that $\tilde{S}_{N, I}[t]$ is product invariant. If $z$ and $\tilde{z}$ are two interpolation points, and $k$ and $\tilde{k}$ two integers such that $z+k=\tilde{z}+\tilde{k}$, then $\left((t+k)^{p}\right)^{(j)}(z)=\left((t+\tilde{k})^{p}\right)^{(j)}(\tilde{z})$. This proves that the sequences $\mathbf{F}_{1}^{-1}\left(t^{p}\right)=\left((t+k)^{p}\right)_{k \in \mathbb{Z}}$ belong to $\tilde{S}_{N, I}[t]$.

Let us prove now the inclusion property. Denote by $T_{l}$ the sequence $\left((t+k)^{l}\right)_{k \in I}, l=0,1$. By assumption, $T_{0} \in \Sigma_{I} \cap \tilde{S}_{N, I}[t]$ and $T_{1} \in \Sigma_{I} \cap \widetilde{S}_{N, I}[t]$; hence the powers $T_{n} \stackrel{\text { def }}{=} T_{n-1} * T_{1}$ belong to $\Sigma_{I} \cap \tilde{S}_{N, I}[t]$. One can prove that any element $p$ of $\tilde{S}_{N, I}[t]$ can be uniquely represented by a polynomial $H^{-1} p$ of $\mathbb{R}_{\Omega_{I}}[t]$, where $\Omega_{I}$ is an integer determined by the overlapping sampling points, and that $H$ commutes with the product. Hence, if $n<\Omega_{I}, T_{n}$ has the unique representation $H^{-1} T_{n}=t^{n}$ in $\mathbb{R}_{\Omega_{I}}[t]$. As a consequence, $\left(T_{0}, T_{1}, \ldots, T_{\Omega_{I}-1}\right)$ is a basis of $\tilde{S}_{N, I}[t]$ because it is the image of the basis $\left(1, t, \ldots, t^{\Omega_{I}-1}\right)$ of $\mathbb{R}_{\Omega_{I}}[t]$. Since $\left(T_{0}, T_{1}, \ldots, T_{\Omega_{I}-1}\right)$ is also in $\Sigma_{I}$, necessarily $\Sigma_{I}$ includes the algebra generated by this family, e.g. $\tilde{S}_{N, I}[t]$.

Theorem 4 indicates that the only degrees of freedom which are left in the definition of an algebra $\Sigma_{I}$ lie in the singularities at the overlapping sampling points.

\subsection{Recovering properties $(P 1)$ and $(P \mathscr{2})$ in the case of a two point interpolation product}

There are as many spaces of regular functions as there are interpolation points. To simplify, we consider here the case where the product is defined by a Hermite interpolation between two points of equal order. We shall assume, without loss of generality, that these two points are 0 and 1 . This implies that the piecewise polynomials are defined by an even number of parameters, e.g., their degree $N$ is odd, e.g. say, $N=2 n+1$. 


\subsubsection{Hermite function representation and interpolation}

After a suitable piecewise polynomial approximation of degree $2 n+1$, functions are represented as sequences of pairs $\left(l_{k}, r_{k}\right)_{k \in \mathbb{Z}}$ of polynomials of degree $n$. The corresponding piecewise polynomial function can be written

$$
\sum_{k \in \mathbb{Z}} \sum_{i=0}^{i=n}\left[l_{k}^{(i)}(0) H_{i}\left(\frac{t}{\delta}-k\right)+r_{k}^{(i)}(0)(-1)^{i} H_{i}\left(\frac{1-t}{\delta}-k\right)\right] 1_{[k, k+1)}\left(\frac{t}{\delta}\right)
$$

where $H_{i}(t)$ is the unique polynomial of degree $2 n+1$ which satisfies

$$
H_{i}^{(j)}(0)=\delta_{i, j} \text { and } H_{i}^{(j)}(1)=0 \text { for } 0 \leq j \leq n
$$

A method for constructing $H_{i}, 0 \leq i \leq n$ can be found in [12]. Repeated applications of Rolle's theorem prove that $H_{i}(t)>0$ if $0<t<1$.

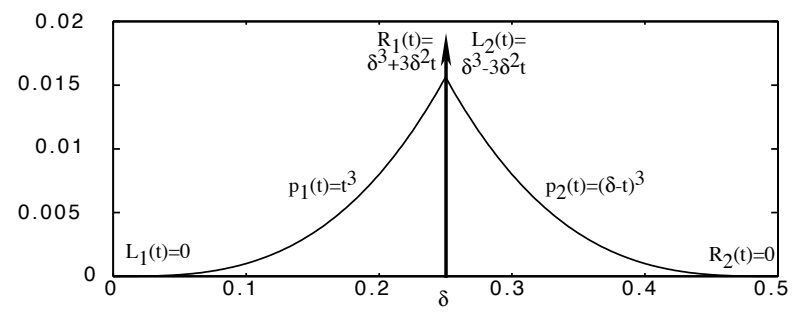

Figure 1: A sequence $\left(p_{1}(t)=t^{3}, p_{2}(t)=(\delta-t)^{3}\right)$ of two cubic polynomials can be viewed as a sequence $\left(\left(L_{1}(t)=0, R_{1}(t)=\delta^{3}+3 \delta^{2} t\right),\left(L_{2}(t)=\right.\right.$ $\left.\left.\delta^{3}-3 \delta^{2} t, R_{2}(t)=(\delta-t)^{3}\right)\right)$ ) of couples of polynomials with degree $1 . L_{i}$ polynomials are Taylor expansions at the left point; $R_{i}$ are expansions at the right point. "Regular functions" are defined by $R_{1}=L_{2}$.

\subsubsection{Product definition}

The product $\left(l_{k}, r_{k}\right) \times \times\left(\lambda_{k}, \rho_{k}\right)$ of two sequences $\left(l_{k}, r_{k}\right)$ and $\left(\lambda_{k}, \rho_{k}\right)$ is defined by

$$
\left(l_{k}, r_{k}\right) \times \times\left(\lambda_{k}, \rho_{k}\right)=\left(l_{k} \times \lambda_{k}, r_{k} \times \rho_{k}\right)
$$

with

$$
(p \times q)(t)=\sum_{i+j \leq n} p_{i} q_{j} t^{i+j} \in \mathbb{R}_{n}[t]
$$




\subsubsection{Recovering properties (P1) and (P2)}

The results of section 3.3 are used to build product invariant subspaces using a regularization operator which removes singularities; $(P 1)$ is thus recovered. The symmetric structure of this operator makes it possible to recover property $\left(P^{2}\right)$.

Regularization operator Subalgebras are defined by removing singularities. Define the regularization operator $\mathbf{R}_{k}$ by

$$
\begin{aligned}
\mathbf{R}_{k}(l, r)_{k} & =\left(l_{k}, \frac{r_{k}+l_{k+1}}{2}\right) \\
\mathbf{R}_{k}(l, r)_{k+1} & =\left(\frac{r_{k}+l_{k+1}}{2}, r_{k+1}\right) \\
\mathbf{R}_{k}(l, r)_{j} & =\left(l_{j}, r_{j}\right) \text { otherwise }
\end{aligned}
$$

All of the $\mathbf{R}_{k}$ 's commute, and, given an index set $I$, the operator $\mathbf{R}_{I}$ is defined as the composition of the $\mathbf{R}_{k}$ 's for $k \in I$. If $S$ is a subalgebra of $S_{N}[t]$, then $\mathbf{R}_{I} S$ is also a subalgebra of $S_{N}[t]$. Moreover, if $S$ includes regular functions, then $\mathbf{R}_{I} S$ also includes regular functions. Finally, $\mathbf{R}_{I}$ reproduces polynomials like in the Strang and Fix conditions (9) because polynomials sequences are regular functions which are unchanged by $\mathbf{R}_{I}$. It is thus a high order approximation projector when the interval between the polynomial samples tends to 0 .

Singularity operator The singularity operator $\mathbf{S}_{k}$ is defined by $\mathbf{S}_{k}=$ Id $-\mathbf{R}_{k}$, e.g.

$$
\begin{aligned}
\mathbf{S}_{k}(l, r)_{k} & =\left(0, \frac{r_{k}-l_{k+1}}{2}\right) \\
\mathbf{S}_{k}(l, r)_{k+1} & =\left(\frac{l_{k}-r_{k+1}}{2}, 0\right) \\
\mathbf{S}_{k}(l, r)_{j} & =(0,0) \text { otherwise }
\end{aligned}
$$

If $I \subset \mathbb{Z}, \mathbf{S}_{I}$ is defined as $\mathbf{S}_{I}=\mathrm{Id}-\mathbf{R}_{I}$.

Recovering properties $(\boldsymbol{P} 1)$ and (P2) These two operators are symmetry based, like the decomposition in the Haar basis. For this reason, properties (P1) and (P2) are recovered: 
Theorem 5 (Product and decomposition) Let $I \subset \mathbb{Z}, \boldsymbol{V}_{I}$ the image of $\boldsymbol{R}_{I}, \boldsymbol{W}_{I}$ the image of $\boldsymbol{S}_{I}$. Then $S_{N}[t]=\boldsymbol{V}_{I} \oplus \boldsymbol{W}_{I}$, and

(P1) the product of two elements of $\boldsymbol{V}_{I}$ is an element of $\boldsymbol{V}_{I}$

(P2) the product of an element of $\boldsymbol{V}_{I}$ with an element of $\boldsymbol{W}_{I}$ is an element of $\boldsymbol{W}_{I}$

(P3) moreover, the product of two elements of $\boldsymbol{W}_{I}$ is an element of $\boldsymbol{V}_{I}$

Proof. The decomposition of $x$ between $\mathbf{R}_{I} x$ and $\mathbf{S}_{I} x$ is the decomposition of a pair of vectors into the symmetric and their antisymmetric parts.

Theorem 5 yields a polynomial formula which is similar to (1):

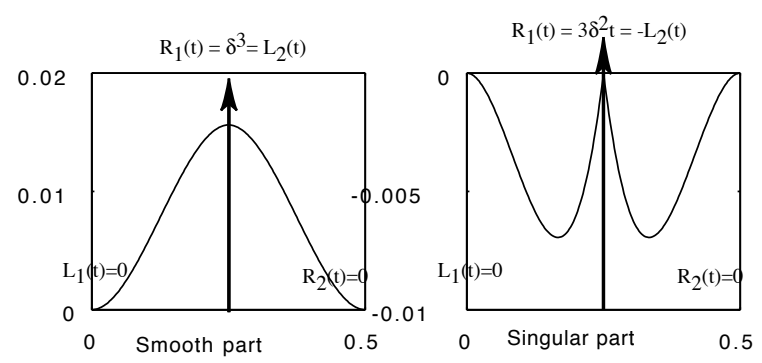

Figure 2: The piecewise polynomial sequence of figure 1 is decomposed by symmetry into its regular part and its singular part.

Corollary 1 If $x \in S_{N}[t]$ and $P \in \mathbb{R}[t]$, then

$$
\boldsymbol{R}_{I}(P(x))=\sum_{k=0}^{k=\infty} \frac{P^{(2 k)}(e)}{2 k !} w^{2 k}
$$

with $e=\boldsymbol{R}_{I} x$ and $w=\boldsymbol{S}_{I} x$, and $P(x)$ is defined with the product operator ${ }^{3}$ *.

\footnotetext{
${ }^{3}$ Actually, $\mathbb{R}$ is identified to the constants of $S_{N}[t]$, e.g., the coefficients of the polynomial $P$ are constant functions.
} 


\subsubsection{Application}

We give a very simple application. We wish to estimate the commutation defect between the polynomial and the regularization (approximation) operator. To do so, we estimate $\mathbf{R}_{I}(P(x))-P\left(\mathbf{R}_{I} x\right)$. This calls for a norm on the elements of $S_{N, \delta}(t)$ which is compatible with the product, e.g. $\|x * y\| \leq\|x\|\|y\|$. Let us represent the elements $\sigma$ of $S_{N, \delta}(t)$ as sequences $\left(l_{k}, r_{k}\right)$ of polynomial pairs of degree less or equal to $n$ and renumber it as a sequence of polynomials $p_{k}$. A $*$ product compatible norm $\|\sigma\|_{*}$ of $\sigma$ is $\sup _{k}\left\|p_{k}\right\|_{n}$, where $\|p\|_{n}$ is the product compatible polynomial norm $\left\|\sum_{i=0}^{i=n} p_{i} t^{i}\right\|_{n}=\sum_{i=0}^{i=n}\left|p_{i}\right| \delta^{i}$. Assuming that the norms of the derivatives $P^{(2 k)}(e)$ are uniformly bounded by $K$, we get the estimate from equation $(10)$ :

$$
\left\|\mathbf{R}_{I}(P(x))-P\left(\mathbf{R}_{I} x\right)\right\|_{*} \leq K\left(\cosh \left\|\mathbf{S}_{I} x\right\|_{*}-1\right)
$$

This estimate can be used to select on which index set $I$ the regularization operator $\mathbf{R}_{I}$ must bear in order to have a commutation defect which is smaller than a given threshold $\epsilon$.

\section{Conclusion}

From a simple averaging scheme we have drawn equation (1) which relates the average of $f(u)$ to the average $E(u)$ of $u$ and the averages of the powers of the remainder $u-E(u)$. A projector satisfies (1) if and only if it satisfies properties $(P 1)$ and $(P \mathscr{2})$. This implies that the product of two "averaged" functions be itself an "averaged" function. This property is very strong; in fact, we show that, when the space of "averaged" functions is generated by the shifts of a single waveform, this property implies that the waveform is the characteristic function of an interval. In terms of approximation theory, this means that the order of approximation provided by the projection on the space of "averaged" functions is the lowest possible.

To work around this limitation, we observe that the space of "averaged" functions is an approximation space and that an approximate product may be substituted to the actual one without degrading the order of approximation. We have exhibited in section 2.2.2 a new result on the characterization of such approximate product operators by means of a Strang and Fix like condition.

However, it seems difficult to construct such approximate products in a general framework. This is why we have restricted ourselves to piecewise 
polynomial functions. For this space of functions we have exhibited a complete parameterization of approximate product operators which is based on Hermite interpolation.

Using such an approximate product implies the existence of a minimal product invariance subspace; it excludes the construction of embedded approximation spaces obtained by rescaling, in the manner of multiresolution approximations. By characterizing the minimum approximation subalgebra, we can observe that the "smooth" part of the function should be obtained by removing the singularities at the overlapping interpolation points.

In the case of a two point interpolation scheme, this idea is explicited in section 3.4.3 and a suitable decomposition of the function into a regular and a singular part is exhibited which satisfies conditions (P1) and (P2). The regularization operator is an approximation projector which satisfies a simplified version of equation (1), which was the goal of the article. This operator is based on the averaging of the left and right derivatives of the function at the interpolation points. As such, it gives sense to the "average" of a nonlinear function $f(u)$ as a function of the input $u$.

This simple decomposition works because it is based on the symmetry properties of its arguments, a structure which is sufficiently strong to be preserved through nonlinearities.

Overall, it is possible to retrieve properties (P1) and (P2) by using approximate product operators on piecewise polynomial approximations of functions. Equation (10) is very simple and can be used, for instance, to detect which singularities are negligible in the commutation between the regularization and the polynomial operator.

\section{A Proof of lemma 1}

Proof. Let $r$ the maximum integer $n$ for which $\phi_{0} * \phi_{n} \neq 0$; because the product is commutative, $r \geq 0$. Let $M_{r}=\max \left\{k \in \mathbb{Z}\right.$ s.t. $\left.C_{k}\left(\varphi_{0} * \varphi_{r}\right) \neq 0\right\}$. Consider now $\left(\phi_{0} * \phi_{r}\right) * \phi_{M_{r}+r}$; there exist a non zero constant $a$ such that it is equal to $a \phi_{M_{r}} * \phi_{M_{r}+r}$, which is itself non zero. On the other hand, it is also equal to $\left(\phi_{0} * \phi_{M_{r}+r}\right) * \phi_{r}$, which is zero if $M_{r}>0$; hence, $M_{r} \leq O$. By a symmetric argument, one verifies that $m_{r}=\min \left\{k \in \mathbb{Z} / C_{k}\left(\varphi_{0} * \varphi_{r}\right) \neq 0\right\}$ satisfies $m_{r} \geq 0$; hence, $m_{r}=M_{r}=0$. This implies that $\phi_{0} * \phi_{r}=A \phi_{0}$. Consider now $\phi_{-r} *\left(\phi_{0} * \phi_{r}\right)=A^{2} \phi_{-r}$; it is also equal to $\left(\phi_{-r} * \phi_{r}\right) * \phi_{0}$, which is non zero if and only if $r=0$. If $*$ is the usual product, taking $x=\phi_{i}$ and 
$y=\phi_{j}$ with $i \neq j$ in (2) implies $C_{n}\left(\phi_{i} \phi_{j}\right)=0$ for all $n$, e.g. $\phi_{i} \phi_{j}=0$ almost everywhere.

The domain argument used in the proof is illustrated in figure 3 .

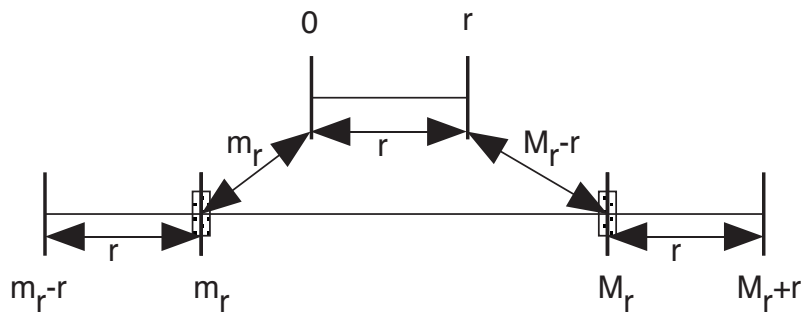

Figure 3: The diffusion of the analysis and reconstruction processes implies a diffusion of the product operator.

\section{B Proof of theorem 2}

Proof. Necessary condition: if $i+j \leq N$ and $(k, l) \in I_{N}$, then $k \geq i+1$ or $l \geq j+1$. Inserting $f(t)=t^{i}$ and $g(t)=t^{j}$ with $i+j \leq N$ in (4) proves, with the Strang and Fix conditions, that (5) holds.

Sufficient condition: Denote by $\mathbf{T} x$ the Taylor expansion of a function $x$ at point $t$ with degree $N$. The left handside of (4) is decomposed as

$$
\begin{aligned}
& \left|f g(t)-\left[\left(E_{\delta} f\right) *_{\delta}\left(E_{\delta} g\right)\right](t)\right| \\
\leq & |f g(t)-\mathbf{T}(f g)(t)| \\
& +\left|\left[E_{\delta} f *_{\delta} E_{\delta}(g-\mathbf{T} g)\right](t)\right| \\
& +\left|\left[E_{\delta}(f-\mathbf{T} f) *_{\delta} E_{\delta} \mathbf{T} g\right](t)\right| \\
& +\left|\mathbf{T}(f g)(t)-\left[E_{\delta}(\mathbf{T} f) *_{\delta} E_{\delta}(\mathbf{T} g)\right](t)\right|
\end{aligned}
$$

The definition of $\mathbf{T} x$ implies that (11) is zero. A localized version of the Strang and Fix approximation conditions and the localization of the product implies that (12) is bounded by a term like (4) with $k=0$ and $l=N+1$. Similarly, (13) is bounded by the sum of two terms with $k=N+1$ and $l=1$ and $l=N+1$. Finally, the Strang and Fix condition $E_{\delta}\left(t^{i}\right)=t^{i}$ and condition (5) imply that (14) is equal to $\left[\mathbf{T}(f g)-\mathbf{T} f *_{\delta} \mathbf{T} g\right](t)=\sum_{(k, l) \in I_{n}} f^{(k)}(t) k ! \times$ $g^{(l)}(t) / l ! \times\left((s-t)^{i} *_{\delta}(s-t)^{j}\right)(t)$ Together with the continuity of the product, this proves that (14) is bounded by a term like in (4). 


\section{Proof of lemma 2}

Proof. Let $T_{N+1}=t^{N} \times t$. There exists a unique family $\left(a_{0}, \ldots, a_{N}\right)$ such that

$$
T_{N+1}=\sum_{i=0}^{i=N} a_{i} t^{i} \stackrel{\text { def }}{=} Q(t)
$$

Let $\left(z_{0}, \ldots, z_{N}\right)$ the complex roots of the (usual) polynomial $t^{N+1}-Q(t)$. Then $T_{N+1}$ is the Hermite interpolation of $t^{N+1}$ at these points. We are going to show that $\times$ is obtained by Hermite interpolation of the usual product at $\left(z_{0}, \ldots, z_{N}\right)$. To do so, we only have to prove it is true for monomials generated by $\times$.

The latter are defined recursively by $T_{N+p}=T_{N+p-1} \times t$. We assume that $T_{N+p-1}$ is the interpolation of $t^{N+p-1}$ at $\left(z_{0}, \ldots, z_{N}\right)$. There also exist a polynomial $q(t)$ of degree $<N$ and a real $b$ such that $T_{N+p-1}=q+b t^{N}$. Then

$$
T_{N+p}=q t+b T_{N+1}
$$

By assumption, $q\left(z_{i}\right)+b\left(z_{i}\right)^{N}=z_{i}^{N+p-1}$; hence,

$$
\begin{aligned}
T_{N+p}\left(z_{i}\right) & =\left(z_{i}^{N+p-1}-b\left(z_{i}\right)^{N}\right) z_{i}+b T_{N+1}\left(z_{i}\right) \\
& =z_{i}^{N+p}-b z_{i}^{N+1}-b z_{i}^{N+1}=z_{i}^{N+p}
\end{aligned}
$$

which proves the interpolation result at the order 0 . For higher orders $k$, the induction becomes:

$$
\begin{aligned}
T_{N+p}^{(k)}\left(z_{i}\right) & =(q t)^{(k)}\left(z_{i}\right)+b T_{N+1}^{(k)}\left(z_{i}\right) \\
& =\left(T_{N+p-1} t-b t^{N+1}\right)^{(k)}\left(z_{i}\right)+b T_{N+1}^{(k)}\left(z_{i}\right) \\
& =\left(T_{N+p-1} t\right)^{(k)}\left(z_{i}\right) \\
& =T_{N+p-1}^{(k)}\left(z_{i}\right) z_{i}+T_{N+p-1}^{(k-1)}\left(z_{i}\right) \\
& =\left(t^{N+p-1}\right)^{(k)}\left(z_{i}\right) z_{i}+\left(t^{N+p-1}\right)^{(k-1)}\left(z_{i}\right) \\
& =\left(t^{N+p}\right)^{(k)}\left(z_{i}\right)
\end{aligned}
$$

Conversely, one verifies that the Hermite interpolation is indeed associative and that, by definition, it preserves the polynomials of degree $\leq N$. 


\section{References}

[1] J. Sanders, F.Verhulst, Averaging Methods in Nonlinear Dynamical Systems, Springer-Verlag, New York, 1985.

[2] I. Daubechies, Ten Lectures on Wavelets, SIAM, Philadelphia, PA, 1992.

[3] Y. Meyer, Wavelets and Operators, Advanced mathematics, Cambridge University Press, 1992.

[4] S. Mallat, A Wavelet Tour of Signal Processing, second ed., Academic Press, 1999.

[5] G. Strang, G. Fix, A Fourier analysis of the finite element variational method, Construct. Aspects of Funct. Anal. (1971) 796-830.

[6] I. Daubechies, Orthonormal bases of compactly supported wavelets, Commun. on Pure and Appl. Math. 41 (1988) 909-996.

[7] G. Beylkin, On the fast algorithm for multiplication of functions in the wavelet bases, in: Proceedings of the International Conference "Wavelets and Applications", Toulouse, 1992.

[8] G. Deslauriers, S. Dubuc, Symmetric iterative interpolation, Constr. Approx. 5 (1989) 49-68.

[9] D. Donoho, Interpolating wavelet transforms, Tech. rep., Department of Statistics, Stanford University (1992).

[10] S. Jaffard, Exposants de hölder en des points donnés et coefficients d'ondelettes, C. R. Acad. Sci. Paris 308 (Série 1) (1989) 79-81.

[11] F. Chaplais, Product invariant piecewise polynomial approximations of signals, available at http://cas.ensmp.fr/ ${ }^{\sim}$ chaplais/FTP/ Preprints/PiecewisePoly.pdf.

[12] J. Stoer, R. Burlisch, Introduction to Numerical Analysis, Texts in Applied Mathematics, Springer-Verlag, New York, 1993. 